\title{
Labyrinthe
}

40 | 2013

Comme les abeilles

\section{Le « monde » des abeilles selon von Uexküll}

\section{Bruce Bégout}

\section{OpenEdition}

Journals

Édition électronique

URL : http://journals.openedition.org/labyrinthe/4309

DOI : $10.4000 /$ labyrinthe.4309

ISSN : 1950-6031

Éditeur

Hermann

Édition imprimée

Date de publication : 1 mars 2013

Pagination : 47-49

ISBN : 9782705688400

\section{Référence électronique}

Bruce Bégout, "Le «monde » des abeilles selon von Uexküll », Labyrinthe [En ligne], 40 | 2013, mis en ligne le 01 mars 2015, consulté le 01 mai 2019. URL : http://journals.openedition.org/labyrinthe/4309 ; DOI : 10.4000/labyrinthe.4309

Propriété intellectuelle 


\title{
Le « monde » des abeilles selon von Uexküll
}

\author{
Bruce BÉGOUT
}

Ce qui surprend dans l'approche de Jakob von Uexküll, c'est son insistance sur la subjectivité de l'animal. Non pas un vulgaire anthropomorphisme: il veut dire que l'animal doit être considéré comme point de référence zéro pour comprendre l'organisation de son monde par lui-même. En ce sens l'animal est un sujet qui produit un monde, suivant un plan d'organisation qui est le sien. C'est récuser l'argument mécaniste ou objectiviste: l'animal est ici considéré comme acteur et auteur de son monde. Un texte très connu, Mondes animaux et monde humain, comprend un passage fameux à propos de la tique; mais il est également d'un grand intérêt lorsqu'il évoque les abeilles (notons au passage que von Uexküll y cite souvent Karl von Frisch) ${ }^{1}$.

Trois types de questions sont posés: elles concernent le milieu; l'accord entre l'abeille et son milieu; la subjectivité.

1) La question du milieu est centrale dans cette approche: pour comprendre un animal, il faut le comprendre d'emblée dans son milieu. Il distingue d'emblée le milieu et l'environnement. Tous les animaux partagent le même monde que nous : c'est ce que von Uexküll nomme Umgebung, «environnement » (qui a aussi été traduit par « entourage »). Celui-ci est un et même pour tous. Seulement l'abeille ne constitue pas de rapport direct à l'environnement: elle lui est au fond indifférente. Elle y sélectionne des éléments à partir desquels elle construit un monde propre à son espèce, un monde générique. C'est son milieu: l'organisation d'un territoire propre autour d'éléments significatifs prélevés sur l'environnement. L'animal ne réagit qu'à des éléments du milieu, ce que von Uexküll nomme le «plan interne » (aujourd'hui l'analyse date sans doute un peu, d'autres notions comme

1. Jakob von Uexküll, Streifzüge durch die Umwelten von Tieren und Menschen (illustrations de Georges Kriszat), Berlin, Springer, 1934; trad. fr. (par Philippe Muller) Mondes animaux et monde humain, Paris, Gonthier, 1965; nouvelle trad. fr. (par Charles Martin-Freville) Milieu animal et milieu humain, Paris, Payot \& Rivages, 2010. 


\section{Labyrinthe, $n^{\circ} 40$}

« instinct » ou « gène » sont peut-être davantage utilisées, mais sur le fond on parle de la même chose). Image célèbre : le dessin de la prairie (environnement) / formes archétypales de ce que l'abeille perçoit (dans son milieu). Au fond l'animal, donc n'a pas d'environnement. Des animaux d'espèces différentes ne partagent pas le même milieu, ils ont chacun un milieu spécifique. Von Uexküll utilise la notion de « cercles fonctionnels » pour nommer la manière dont l'animal tire de son «plan interne » différentes fonctions qui organisent le milieu. Ces besoins sont de quatre types concernant les abeilles (comme pour d'autres hyménoptères): construction et conservation de la demeure; alimentation; combat contre les animaux hostiles; sexualité. Ces fonctions sélectives sont les éléments de construction du monde de l'abeille. Celle-ci vit donc dans quatre espaces différents : sa demeure, son territoire immédiat (qui n'est pas le milieu), le milieu, et l'environnement physique commun à tous. Les cercles fonctionnels sont ce qui découpe l'environnement en milieux : purement subjectifs, au sens où c'est l'animal qui est le sujet de son milieu. L'abeille configure son monde.

2) Le deuxième point concerne la relation entre les besoins que l'animal porte en lui sur son «plan interne » et le milieu : ce qui pose la question de la pré-harmonie entre eux. Von Uexküll s'étonne de ce qu'il norme avec un ensemble de métaphores musicales: d'abord «mélodie », puis « duo », puis « structure contrapuntique ». Cela donne un très beau passage sur le «monde contrapuntique » de l'abeille: « si on les examine bien les instruments de l'orchestre présentent dans leur structure même un comportement contrapuntique [il vient de parler de Bach et du contrepoint chez Bach]. Cela apparaît avec une netteté particulière dans un orchestre naturel comme celui qu'offre une prairie. Il nous suffit de penser à la fleur située dans nos quatre milieux [référence à un point un peu secondaire ici]. Le plus frappant est le rapport entre la structure de la fleur et la structure de l'abeille, rapport dont on peut dire: si la fleur n'était faite pour l'abeille, si l'abeille n'était faite pour la fleur, jamais elles ne seraient à l'unisson. » Voilà énoncée en même temps la proposition fondamentale de toute technique naturelle. Nous y reconnaissons la sagesse de Goethe qui écrit: « si l'œil n'était fait pour le soleil jamais il ne pourrait le regarder, et si le soleil n'était fait pour l'œil jamais il ne brillerait en aucun ciel ». L'idée est bien celle d'une pré-harmonie entre la structure 


\section{Le « monde » des abeilles selon von Uexküll}

physiologique de l'abeille et son monde, sans qu'on puisse dire ce qui a préconfiguré quoi. C'est cet accord pré-établi entre l'organisme et son milieu que von Uexküll nomme structure contrapuntique. Il veut éviter une compréhension purement éthologique, où le comportement serait expliqué comme une réaction au milieu, et le milieu comme réaction au comportement. Pour von Uexküll c'est une compréhension causale dont il faut sortir sans pour autant verser dans le finalisme. C'est essentiellement l'abeille qui est mobilisée pour poser cette compréhension contrapuntique.

3) Revenons à la notion de subjectivité. Que veut-il dire par là ? D'abord le refus de l'objectivité : l'animal n'a pas face à lui des données objectives qu'il percevrait. L'animal n'a pas de rapport à l'objectivité. L'abeille ne voit pas la fleur en tant que telle, mais déjà en tant que porteur de valeur (cf. merkmal chez Heidegger) ou de signification - ce qu'on nomme parfois « valence ». Elle fait du perspectivisme nietzschéen; c'est la vie qui interprète en elle, et elle interprète tout à travers ce plan interne des besoins.

4) Un second aspect dérive de l'harmonie pré-établie: dès qu'il y a un stimulus significatif pour l'abeille, elle y répond, et dès qu'elle adopte un comportement c'est dans l'attente des éléments correspondants du milieu. Mais von Uexküll remarque qu'il peut dans certains cas se produire l'un sans l'autre: un comportement de l'animal sans objet. Ainsi dans le cas (cité par Heidegger) où l'on sectionne l'abdomen de l'abeille: elle ingurgite alors du pollen sans s'arrêter, faute de satiété. Remplissage à vide de l'instinct qui n'est jamais comblé par une réponse définitive. Au fond, il y a parfois une forme d'inventivité : pour trouver un élément de substitution lorsque l'élément attendu du milieu n'est pas là. (C'est ce que reprend Lorentz dans son étude des instincts sociaux des animaux : l'instinct maternel de l'oie est vide d'objet, structure maternelle que n'importe quel objet de substitution peut remplir.) L'animal est un individu qui apporte avec lui une structure d'ouverture du monde. 